\title{
Investigating recombinant protein exudation from roots of transgenic tobacco
}

\author{
Francesca PIZZUTI and Lorenza DARODA* \\ ENEA-Casaccia Research Center, BAS-BIOTECGEN Rome, Italy
}

It is widely acknowledged that plant-made pharmaceuticals (PMPs) offer numerous benefits, including inexpensive production, biological safety and the facility for production at agricultural scale. At the same time, it is important to minimize any potential risk associated with this new technology, including the potential release of bioactive proteins into the environment. To address this issue, we studied transgenic Nicotiana benthamiana and Nicotiana tabacum plants expressing two recombinant single-chain variable fragment (scFv) antibodies, respectively scFvB9 and ScFvH10. ScFvB9 was raised against glycoprotein G1 of Tomato spotted wilt virus (TSWV), and ScFvH10 was raised against human tumor-associated antigen tenascin-C. Both antibodies were targeted to the secretory pathway using the $\mathrm{N}$-terminal signal peptide from Phaseolus vulgaris polygalacturonaseinhibiting protein (PGIP), and ScFvH10 carried in addition a C-terminal KDEL tetrapeptide for retention in the endoplasmic reticulum (ER). Sterile hydroponic cultures were established, allowing us to investigate whether scFvB9 and scFvH10 were present in root exudates. Intercellular fluids extracted from different plant tissues were analyzed by western blotting revealing the presence of scFvB9. Successful secretion of scFvB9 in hydroponic medium was also demonstrated, whereas no scFvH10 could be detected in the leaf, stem or root apoplast, nor secreted into the hydroponic medium. Our results show that scFvH10 release or diffusion from the roots of transgenic plants was not occurring, suggesting that the KDEL signal might contribute to the environmental biosafety of crops producing PMPs.

Keywords: transgenic tobacco / recombinant antibody / hydroponic culture / KDEL / rhizosecretion / plant molecular farming / biosafety

\section{INTRODUCTION}

The use of transgenic plants for producing pharmaceutical substances (plant molecular farming) is a growing business, confirmed by the increasing number of companies that are investing in this field worldwide. Plants, seeds and cultured plant cells are potentially one of the most economical systems for large-scale production of recombinant proteins for the biochemical, veterinary and pharmaceutical industries (Boehm, 2007; Floss et al., 2007; Kermode, 2006; Pujol et al., 2007; Streatfield, 2007; Twyman et al., 2005). The well-established current state of plant molecular farming is in fact contributing to the extensive development and wide acceptance of this attractive and powerful technology. However, plant made pharmaceuticals (PMPs) must satisfy numerous regulatory requirements before commercialization, not only for the quality and safety of the medical product, but also for the correct management, rigorous confinement and isolation measures of the genetically modified plant chosen as production system (Sparrow et al., 2007; Spök, 2007).

\footnotetext{
* Corresponding author:

lorenza.daroda@casaccia.enea.it
}

Yet, considerable concerns have been raised over the potential impacts of pharmaceutical crops and their bioactive products on agriculture and the environment. The effects of PMP release or dispersal are in fact among the major environmental concerns, since the excessive concentration of toxins or other pharmaceutical products could cause "protein pollution" of the soil environment (Commandeur et al., 2003). This could represent a hazard for soil and rhizosphere microbial communities, as well as for non-target organisms. The magnitude and characteristics of the hazard would however depend on several complex factors, including the nature of the substances, whether it would be fine chemicals, toxins, industrial enzymes, allergens, hormones, vaccines or antibodies. Moreover, most proteins are digestible and nontoxic, but could conceivably be harmful in higher doses or persist longer in the soil (Elbehri, 2005). Beyond the hazards of the novel protein substance, a broad range of other factors have to be considered as well when assessing the environmental risk of plant expression systems, e.g. host plant species, the way plants are cultivated, the land used for cultivation, the way it is monitored (Ramessar et al., 
2007; Wolt et al., 2007). Several research groups have explored the ecological implications and changes arising from transgenic technologies, or investigated innovative and combined approaches for environmental risk assessments (Lilley et al., 2006). Studies on the possible effects on soil ecosystem, microbial communities and nontarget organisms of transgenic plants expressing different traits, including bio-molecules for industrial purposes, have been recently compiled and reported (Sanvido et al., 2007; Widmer, 2007). Researchers have examined different traits and different transgenic plants grown in laboratories, in glasshouses and in open fields. In some cases, when comparing soils associated with control and transgenic plants, differences were identified among microbial communities, but these differences were not related to any effect, since no effects on the rhizosphere were found.

Earlier studies had evaluated the release of proteins from transgenic Bacillus thuringensis $(B t)$ plants. The presence in the soil of $B t$ toxins (Cry proteins) was reported for many diverse $B t$ corn hybrids in different growing conditions of soil environment or sterile hydroponic conditions (Saxena et al., 2002). Other transgenic $B t$ plants such as rice, potato, oilseed rape, tobacco and cotton have also been examined, but the release of $B t$ toxins was reported only for transgenic rice and potato (Saxena et al., 2004). Bt toxins were supposed to enter the soil system via root exudates, residues of plant material, damaged or cast-off root cells. Recent studies examined the release of recombinant proteins in root exudates of transgenic tobacco plants (Nicotiana tabacum L.) expressing human serum albumin (HSA), $\beta$-glucuronidase (GUS), green fluorescent protein (GFP) and glycoprotein B of human cytomegalovirus (gB) (Sabharwal et al., 2007). The presence of HSA and not any other recombinant protein was reported in the root exudates of these plants, yet the reasons for these unexpected differences were not explained. The dissimilarities and high variability encountered in previous studies suggested that further and dedicated investigations are still needed for a complete understanding of root exudation systems, and in particular for field-grown transgenic plants producing PMPs.

For this reason, we have studied exudation from roots of transgenic $N$. tabacum and $N$. benthamiana plants transformed to express recombinant single-chain variable fragment antibodies, respectively scFvB9 and scFvH10. ScFvB9 (raised against the glycoprotein G1 of Tomato spotted wilt virus (TSWV)) and scFvH10 (raised against the human tumor-associated antigen tenascin-C) were both directed to the plant secretory pathway, although scFvH10 carried, in addition, a C-terminal KDEL signal for retention in the endoplasmic reticulum (ER). Root intracellular and extracellular expression of scFvB9 and scFvH10 were examined in detail. Sterile hydroponic cultures of transgenic plants were established to investigate root exudation of scFvB9 and scFvH10 antibodies.

\section{RESULTS}

\section{Analysis of leaf and root expression of scFvB9}

Transgenic $N$. benthamiana plants transformed to express recombinant single-chain scFvB9 antibody were analyzed in our studies (Franconi et al., 1999). As shown in Figure 1A, scFvB9 antibody was expressed under the control of the Cauliflower mosaic virus (CaMV) 35S promoter, targeted to the secretory pathway by means of the N-terminal PGIP leader peptide, and C-terminal c-myc-tagged for purification and immunodetection. Leaf and root tissues harvested from $N$. benthamiana transgenic line 4.2B9 (Fig. 1C) were used for extraction of total soluble proteins (TSP), intercellular fluid soluble proteins (IF) and intracellular proteins (IP). These extracts were analyzed by western blotting using a polyclonal antibody A318 raised against scFvB9, as no detection was observed using anti-c-myc monoclonal antibody 9E10, probably due to the proteolytic cleavage of the c-myc tag (van Esse et al., 2006). A specific immunoreactive band of approximately $30 \mathrm{kDa}$, corresponding to scFvB9 antibody, was clearly revealed in all IF extracts (Fig. 1B), demonstrating that scFvB9 antibody was present in the leaf and root apoplast, the space between the plant cell membrane and the cell wall. Our results confirmed the efficacy and appropriate choice of the PGIP leader peptide for successful targeting of scFvB9 antibody to the secretory pathway. Functionality of leafexpressed scFvB9 was demonstrated in enzyme-linked immunosorbent assay (ELISA) using TSWV (Franconi et al., 1999).

\section{Analysis of leaf, stem and root expression of ScFvH10}

Transgenic N. tabacum cv. Petit Havana SR1 lines pBSK1 and pBSK17, and N. tabacum cv. Mammoth pBSK49 plants transformed to express recombinant single-chain scFvH10 antibody were also analyzed. Like scFvB9, scFvH10 was identically targeted to the secretory pathway and c-myc-tagged for immunodetection, but additionally tagged with a C-terminal KDEL signal for ER retention as shown in Figure 2A. Leaf TSP, IF and IP extracts from pBSK1, pBSK17 and pBSK49 transgenic lines were examined by western blotting using anti-cmyc monoclonal antibody 9E10 (Figs. 2C and 2D, upper panel). The presence of scFvH10 antibody was not revealed in all IF extracts of these different transgenic 

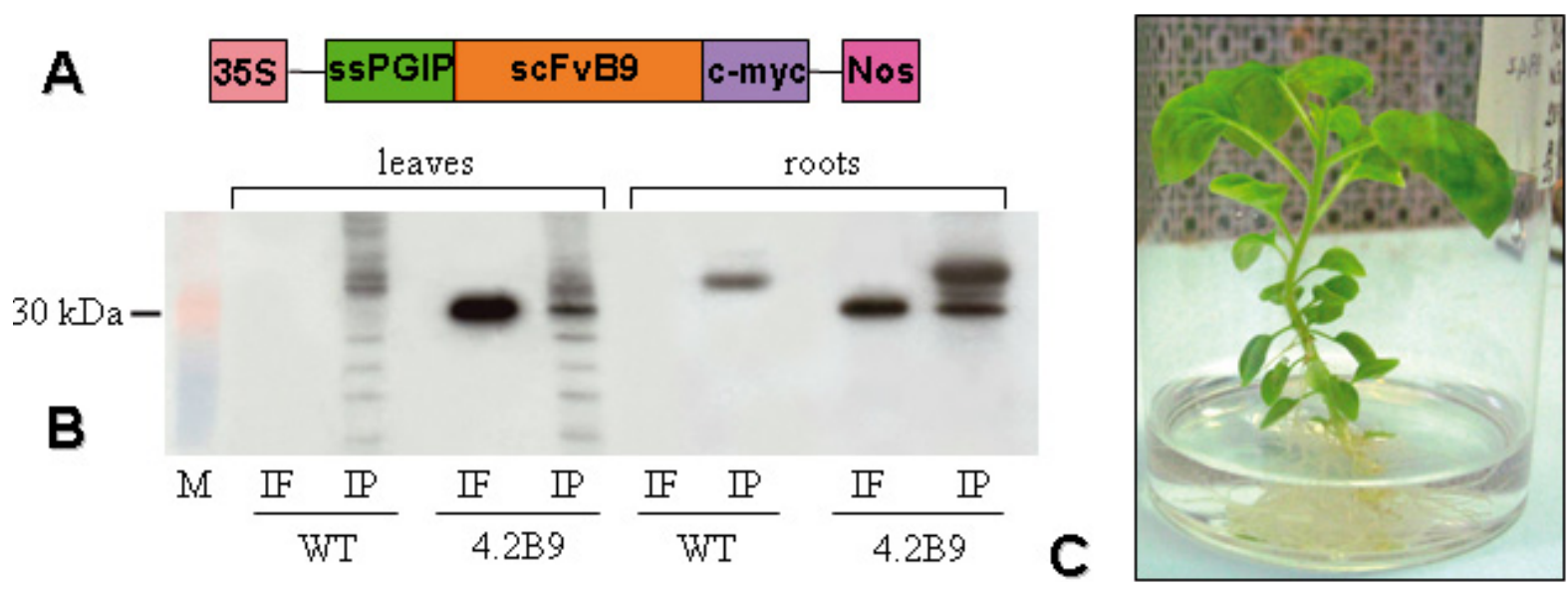

Figure 1. Plant expression of scFvB9. (A) Schematic representation of scFvB9 expression cassette used for plant transformation; 35S: Cauliflower mosaic virus (CaMV) 35S promoter; ssPGIP: signal peptide from the naturally-secreted polygalacturonaseinhibiting protein of Phaseolus vulgaris; c-myc: c-myc tag; Nos: terminator of nopaline synthase gene. (B) Western blotting analysis on intercellular fluid soluble proteins (IF) and intracellular proteins (IP) extracted from leaves and roots of wild-type (WT) and transgenic $N$. benthamiana line 4.2B9, performed using A318 polyclonal antibody. (C) In vitro hydroponic culture of $N$. benthamiana transgenic line 4.2B9. M: molecular weight marker; IF (whole volume of collected extracts); IP (15 $\mu \mathrm{g}$ ).

lines, indicating that the targeting to leaf apoplast of this antibody was prevented. To the contrary, intracellular expression of scFvH10 antibody was clearly demonstrated by the positive immunoreactive band present in all leaf IP extracts at approximately $30 \mathrm{kDa}$ (Figs. 2C and 2D upper panel). These results were subsequently confirmed by a second western blotting analysis in which immunodetection was performed using rProtein L-HRP, which binds specifically to $\mathrm{scFv}$ fragments (Fig. 2D lower panel). Also in this case, no detection was observed in IF extracts, demonstrating that scFvH10 antibody was not targeted to the leaf apoplast. No proteolytic cleavage of the c-myc tag was observed. Affinity and functionality of leaf-expressed scFvH10 antibody was demonstrated by surface plasmon resonance analysis (BIAcore) using human recombinant tenascin-C (data not shown).

IP and IF extracts deriving from stem and root tissues of the same plants were also analyzed by western blotting using anti-c-myc monoclonal antibody 9E10 (Fig. 2E). As previously observed in leaves, the presence of scFvH10 antibody was revealed only in the intracellular protein extracts of stem and root tissues. These results were confirmed performing a second western blotting analysis on the same (stem and root) IP and IF extracts, using rProtein L-HRP. Also in this case, no detection was observed in all IF extracts, demonstrating that scFvH10 antibody was not targeted to the stem and root apoplast (data not shown). Our results demonstrated that in the presence of a C-terminal KDEL signal, no secretion of scFvH10 antibody was occurring in leaf, stem and root apoplast.

\section{Root exudation analysis}

The release of scFvB9 antibody in root exudates of $N$. benthamiana transgenic line $4.2 \mathrm{~B} 9$ was investigated over time under sterile hydroponic conditions. Identical aliquots of culture medium were collected at specific time points during a period of 10 days, and analyzed by western blotting using polyclonal A318 antibody. The presence of scFvB9 antibody could be detected $24 \mathrm{~h}$ after the starting point of the time course and until the last day of the time course (Fig. 3A).

Protease inhibitors or other protein stabilizers were not added to the medium, since during the entire period of 10 days, no degradation of scFvB9 antibody was observed, indicating that the culture conditions were appropriate for rhizosecretion. Cultures were maintained until the 23rd day, and still no degradation of ScFvB9 was observed, unlike what was observed for scFvB9 purified from $E$. coli, which when added to sterile culture medium of a wild-type plant, showed rapid degradation (data not shown). These findings suggest that the scFvB9 rhizosecreted from $N$. benthamiana roots had higher stability. Our results also showed that the PGIP leader peptide was efficient for stable production of scFvB9 antibody in hydroponic conditions.

Adopting the same experimental conditions, we investigated root exudation of $\mathrm{scFvH} 10$ antibody from transgenic $N$. tabacum cv. Mammoth line pBSK49 plant (Fig 3B). The release of scFvH10 antibody was monitored for an identical time period of 10 days, and analyzed by western blotting using anti-c-myc monoclonal 


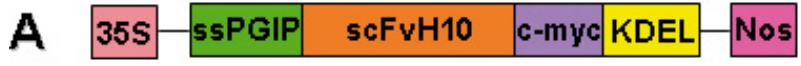
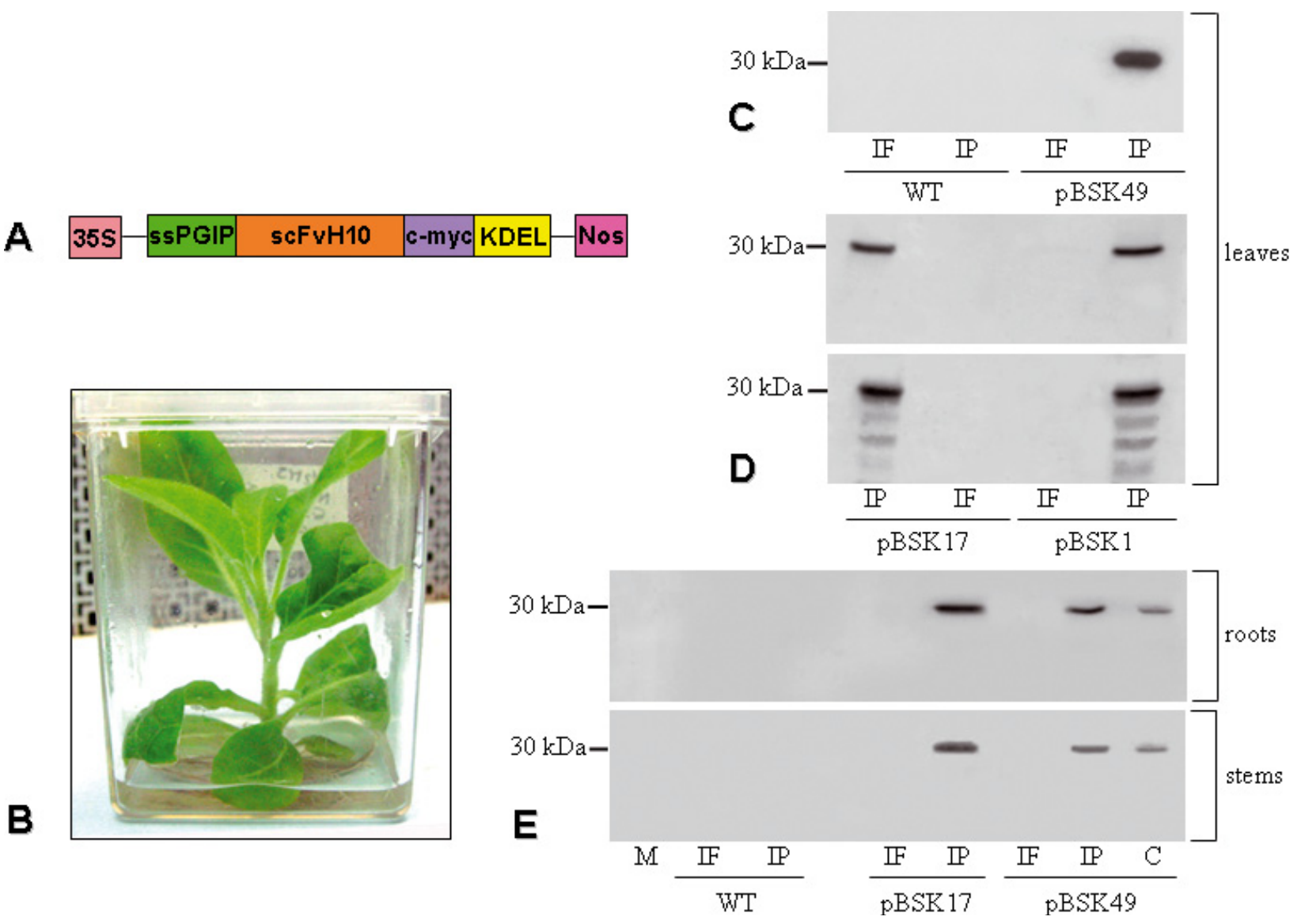

Figure 2. Plant expression of scFvH10. (A) Schematic representation of scFvH10 expression cassette used for plant transformation; 35S: Cauliflower mosaic virus (CaMV) 35S promoter; ssPGIP: signal peptide from the naturally secreted polygalacturonaseinhibiting protein of Phaseolus vulgaris; c-myc: c-myc tag; KDEL: endoplasmic reticulum retention signal; Nos: terminator of nopaline synthase gene. (B) In vitro hydroponic culture of $N$. tabacum cv. Mammoth line pBSK49. (C, D, E) Western blotting analysis on intercellular fluid soluble proteins (IF) and intracellular proteins (IP) extracted from leaves, stems and roots of wild-type (WT), transgenic $N$. tabacum cv. Mammoth pBSK49, transgenic N. tabacum cv. Petit Havana SR1 line pBSK1 and pBSK17, performed using anti-c-myc monoclonal antibody 9E10 and rProtein L-HRP (D lower panel). M: molecular weight marker; C: TSP from leaf of pBSK49 (10 $\mu \mathrm{g})$; IF (whole volume of collected extracts); IP (15 $\mu \mathrm{g})$.

antibody 9E10. Our results showed that during the entire period of the time course no scFvH10 was detected in the culture medium of transgenic $N$. tabacum plants, indicating no accidental release or diffusion of scFvH10 was occurring in root exudates. These findings indicate that the KDEL signal was effective in preventing secretion of scFvH10 in the roots of transgenic tobacco plants.

\section{DISCUSSION AND CONCLUSIONS}

Genetically modified crops have been one of the most rapidly adopted technologies in the history of agriculture, and they have recently been successfully exploited for the expression of important industrial and pharmaceutical products. In addition, the plant secretory pathway, providing correct protein folding, modification, transport and sorting, can offer innovative and low-cost methodology for the large scale production of recombinant pharmaceutical proteins (Faye et al., 2005; Liénard et al., 2007; Saint-Jore-Dupas et al., 2007; Vitale et al., 2005). Yet the implementation of this technology depends on many complex factors not only related to plant production and sorting mechanisms, but also to appropriate assessment of the environmental risks and development of regulatory guidelines (Sparrow et al., 2007). In this regard, data obtained from past model studies, such as with $B t$ crops, seem insufficient to assess the present risks associated 


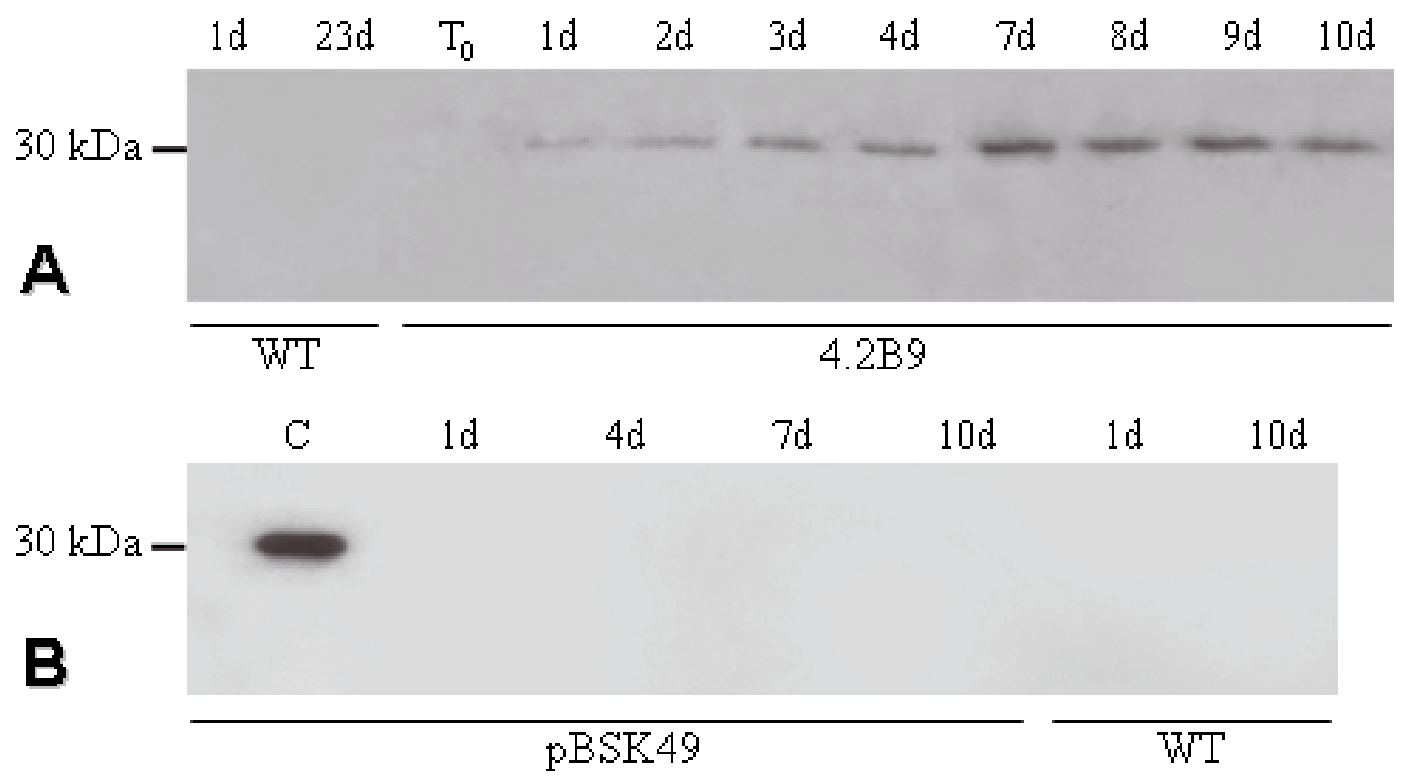

Figure 3. Root exudation in hydroponic medium. Time course performed on aliquots of medium collected at different time points from hydroponic cultures of wild-type (WT), transgenic N. tabacum cv. Mammoth line pBSK49, and transgenic N. benthamiana line 4.2B9, analyzed by western blotting using A318 polyclonal antibody (A) and anti-c-myc monoclonal antibody 9E10 (B). C: TSP from leaf of pBSK49 (10 $\mu \mathrm{g})$; d: days; $\mathrm{T}_{0}$ : starting point.

with plant molecular farming technology, which might need a continuous and case-by-case type of evaluation. Recent studies have shown that protein-trafficking machinery in plants is in fact a very complex, plant-specific and tissue-specific mechanism (Drakakaki et al., 2006; Jurgens, 2004) that needs to be carefully assessed before drawing general conclusions on its safe use in the environment. Therefore, additional studies are needed for the complete understanding, and profitable and safe exploitation of the plant secretory pathway in respect of the environment, in particular for field-grown pharma crops.

For this purpose we investigated the fate of two different recombinant single-chain antibodies, scFvB9 and $\mathrm{scFvH} 10$, targeted to the secretory pathway in transgenic Nicotiana plants. Their expression was analyzed in different tissues of the transgenic plants, with particular attention to roots. We evaluated if scFvB9 and scFvH10 were effectively secreted from roots, and verified the functionality in root tissues of specific signal sequences chosen to regulate and target their expression. Our results showed that $\mathrm{scFvB} 9$ antibody was successfully targeted to the secretory pathway by means of the PGIP leader peptide, expressed in the apoplast, and secreted in hydroponic culture medium in a stable and continuous manner, showing the functionality and applicability of PGIP in rhizosecretion technology (Komarnytsky et al., 2006).

In parallel, we observed that with the same PGIP leader peptide, but in the presence of an additional KDEL signal, transport of scFvH10 antibody to the apoplast was prevented. This occurred in the apoplastic space of leaves, stems and roots of transgenic N. tabacum Petit Havana cv. SR1 and N. tabacum cv. Mammoth plants, in which no accumulation of KDEL-tagged scFvH10 was revealed. Moreover, the presence of scFvH10 was not detected in the culture medium of transgenic $N$. tabacum cv. Mammoth hydroponic culture, indicating that $\mathrm{scFvH} 10$ antibody was not released in the root exudates of transgenic N. tabacum plants. Although studies performed on seeds revealed unexpected dissimilarities (Arcalis et al., 2004; Petruccelli et al., 2006; Van Droogenbroeck et al., 2007), we demonstrated that in tobacco roots, the expression of scFvH10 remains intracellular, and that the KDEL signal is functional. To the best of our knowledge, this is the first time that the intracellular fate of a KDELtagged recombinant single chain antibody was reported in root tissues of transgenic plants.

Moreover, root exudation of recombinant proteins from transgenic tobacco plants was reported for HSA expressed under the constitutive CaMV $35 \mathrm{~S}$ promoter (Sabharwal et al., 2007). In our studies, under the same promoter but with additional targeting mediated by appropriate signal sequences and sorting to specific compartments, we demonstrated that unintended diffusion in the environment of recombinant antibody did not occur. Our results showed that a KDEL-tagged recombinant single-chain antibody in transgenic tobacco roots was neither rhizosecreted nor released by a diffusion-based process into the environment, suggesting that $\mathrm{scFvH} 10$ 
dispersal from the plant's secretory pathway, via root exudates or leakage deriving from the damage of the plant root tissues, did not represent a relevant risk for the environment.

Although the KDEL tag appears to have attractive advantages in containing inadvertent protein secretion, no KDEL-tagged protein production systems in plants have yet been approved by FDA or EMEA. Our data, eventhough not exhaustive, provide information on KDEL functionality in root tissues of tobacco plants that, to our knowledge, was missing from previous studies. We demonstrated that KDEL tag signal sequences might be used to prevent exudation of bioactive proteins in the environment, particularly for crops producing PMPs. Further experiments will be conducted to verify that KDEL secretion-inhibiting sequences are an appropriate tobacco biosafety system.

\section{MATERIALS AND METHODS}

\section{Plant material}

Wild-type and transgenic $N$. benthamiana plants transformed to express recombinant single chain variable fragment antibody scFvB9 directed against TSWV glycoprotein G1 (Franconi et al., 1999) were kindly provided by Dr. R. Franconi (ENEA, Casaccia).

Wild-type and transgenic N. tabacum cv. Petit Havana SR1 and cv. Mammoth plants transformed to express scFvH10 antibody, directed against the human tumorassociated antigen tenascin-C were kindly provided by Dr. M. Donini and Dr. ME. Villani (ENEA, Casaccia).

Both scFvB9 and scFvH10 antibodies were expressed under the control of the CaMV $35 \mathrm{~S}$ promoter, and targeted to the secretory pathway by means of the $\mathrm{N}$-terminal signal peptide (29-aminoacid) derived from the naturally secreted polygalacturonase-inhibiting protein (PGIP) of Phaseolus vulgaris (Toubart et al., 1992). In addition, a KDEL tetrapeptide was introduced at the C-terminal of scFvH10 for antibody retention in the endoplasmic reticulum (ER) (Munro and Pelham, 1987).

\section{Hydroponic growing conditions}

Seeds of N. tabacum cv. Petit Havana SR1 wild-type and transgenic lines (pBSK1 and pBSK17), N. tabacum cv. Mammoth wild-type and transgenic line pBSK49, $N$. benthamiana wild-type and transgenic line 4.2B 9 were surface sterilized by immersion in $70 \%(\mathrm{v} / \mathrm{v})$ ethanol for $2 \mathrm{~min}$, followed by $10 \mathrm{~min}$ immersion in $20 \%$ (v/v) sodium hypochlorite, and rinsed in sterile distilled water. The sterilized seeds were germinated in vitro on half-strength MS basal medium (Murashige and
Skoog, 1962) (Sigma-Aldrich) supplemented with $2 \%$ sucrose and $100 \mu \mathrm{g} . \mathrm{mL}^{-1}$ kanamycin for transgenic material. Seedlings were allowed to reach a length of $5 \mathrm{~cm}$ and then transferred to Magenta boxes (Sigma-Aldrich) containing $25 \mathrm{~mL}$ of sterile liquid half-strength MS medium (as above). Plants were grown aseptically for 90 days at $24{ }^{\circ} \mathrm{C}$ under a $16 / 8 \mathrm{~h}$ day/night photoperiod.

\section{Protein extraction}

Leaf, root, and stem tissues $(0.2 \mathrm{~g})$ were harvested from wild-type and transgenic plants, and frozen in liquid nitrogen. Total soluble proteins (TSP) were extracted in $300 \mu \mathrm{L}$ cold $1 \times$ PBS $(150 \mathrm{mM} \mathrm{NaCl}, 2 \mathrm{mM}$ $\mathrm{NaH}_{2} \mathrm{PO}_{4} \cdot \mathrm{H}_{2} \mathrm{O}, 5 \mathrm{mM} \mathrm{Na}_{2} \mathrm{HPO}_{4} \cdot 12 \mathrm{H}_{2} \mathrm{O}, \mathrm{pH}$ 7.2) containing protease inhibitors (Complete, EDTA-free, Roche). After centrifugation at $16000 \times g$ for $30 \mathrm{~min}$ at $4{ }^{\circ} \mathrm{C}$, supernatants (approximately 300-400 $\mu \mathrm{L}$ ) were recovered for analysis. Total protein concentration was determined by Bradford dye-binding assay (BioRad, Hercules, CA, USA) (Bradford, 1976), using BSA as a standard.

Extraction of intercellular soluble protein fluids (IF) was performed on $0.2 \mathrm{~g}$ of leaf, root and stem tissues as previously described (Franconi et al., 1999). To recover the intracellular fraction (IP), the tissues used for IF extraction were frozen in liquid nitrogen after centrifugation, homogenized and subjected to protein extraction in cold $1 \mathrm{X}$ PBS as described above.

\section{Hydroponic medium analysis}

Time course experiments were performed for a specific number of days: 10 days for wild-type and transgenic $N$. tabacum plants, and 23 days for wild-type and transgenic $N$. benthamiana plants. During this time, $500 \mu \mathrm{L}$ aliquots of sterile in vitro hydroponic culture medium of transgenic and wild-type plants were collected and concentrated $(25 \mathrm{X})$ using a SpeedVac concentrator (Eppendorf Concentrator 5301) at room temperature, until a final volume of $20 \mu \mathrm{L}$, and analyzed as described below.

\section{SDS-PAGE and western blotting}

All protein extracts $(15 \mu \mathrm{g})$ were separated by $12 \%$ SDSPAGE according to Laemmli (1970), using ColorBurst ${ }^{\mathrm{TM}}$ molecular weight marker (Sigma-Aldrich). After electrophoresis, proteins were electrophoretically transferred to PVDF membrane (Millipore) according to Towbin et al. (1979). Membranes were blocked $2 \mathrm{~h}$ at room temperature with $10 \%(\mathrm{w} / \mathrm{v})$ non-fat dry milk in PBS-T (1 X PBS containing $0.1 \%(\mathrm{v} / \mathrm{v})$ Tween 20$)$ and washed thrice for $10 \mathrm{~min}$ in PBST, PBST $+1 \mathrm{M} \mathrm{NaCl}$ and PBST. 
ScFvH10 detection was performed using primary antibody mAb 9E10 (Sigma-Aldrich) (1:3000 dilution) for $2 \mathrm{~h}$ at room temperature in PBST containing 3\% (w/v) non-fat dry milk, followed by $1 \mathrm{~h}$ with HRP-conjugated goat anti-mouse IgG antibodies (GE Healthcare) (1:5000 dilution). ScFvH10 detection was also performed using Immunopure ${ }^{\circledR}$ rProtein L-HRP (Pierce) (1:1000 dilution) for incubation in PBS-T containing 2\% (w/v) BSA. ScFvB9 detection was performed using polyclonal antibody A318 (1:6000 dilution) kindly provided by Istituto Virologia Vegetale (CNR, Torino, Italy) for $2 \mathrm{~h}$ at room temperature in PBST containing 2\% (w/v) BSA, followed by $1 \mathrm{~h}$ with HRP-conjugated anti-rabbit polyclonal antibodies (GE Healthcare) (1:15000 dilution). Peroxidase activity was determined, for both conditions, by enhanced chemiluminescence (ECL Plus ${ }^{\mathrm{TM}}$, GE Healthcare).

\section{ACKNOWLEDGEMENTS}

We are grateful to ENEA researchers for providing plants and reagents, in particular to Dr. Rosella Franconi for motivating us.

Received January 24, 2008; accepted August 24, 2008.

\section{REFERENCES}

Arcalis E, Marcel S, Altmann F, Kolarich D, Drakakaki G, Fischer R, Christou P, Stoger E (2004) Unexpected deposition patterns of recombinant proteins in post-endoplasmic reticulum compartments of wheat endosperm. Plant Physiol. 136: $3457-3466$

Boehm R (2007) Bioproduction of therapeutic proteins in the $21^{\text {st }}$ century and the role of plants and plant cells as production platforms. Ann. N. Y. Acad. Sci. 1102: 121-134

Bradford MM (1976) A rapid and sensitive method for the quantification of micrograms quantities of protein utilizing the principle of protein dye binding. Anal. Biochem. 72: 248-254

Commandeur U, Twyman M, Fisher R (2003) The biosafety of molecular farming in plants. AgBiotechNet 5: 1-9

Drakakaki G, Marcel S, Arcalis E, Altmann F, GonzalezMelendi P, Fischer R, Christou P, Stoger E (2006) The intracellular fate of a recombinant protein is tissue dependent. Plant Physiol. 141: 578-586

Elbehri A (2005) Biopharming and the food system: examining the potential benefits and risks. Agbioforum 8: 18-25

Faye L, Boulaflous A, Benchabane M, Gomord V, Michaud D (2005) Protein modifications in the plant secretory pathway: current status and practical implications in molecular pharming. Vaccine 23: 1770-1778
Floss DM, Falkenburg D, Conrad U (2007) Production of vaccines and therapeutic antibodies for veterinary applications in transgenic plants: an overview. Transgenic res. 16: 315-332

Franconi R, Roggero P, Pirazzi P, Arias FJ, Desiderio A, Bitti O, Pashkoulov D, Mattei B, Bracci L, Masenga V, Milne RG, Benvenuto E (1999) Functional expression in bacteria and plants of an $\mathrm{scFv}$ antibody fragment against tospoviruses. Immunotechnology 4: 189-201

Jurgens G (2004) Membrane traffiking in plants. Annu. Rev. Cell Dev. Biol. 20: 481-504

Kermode AR (2006) Plants as factories for the production of biopharmaceutical and bioindustrial proteins: lessons from cell biology. Can. J. Bot. 84: 679-694

Komarnytsky S, Borisjuk N, Yakoby N, Garvey A, Raskin I (2006) Cosecretion of protease inhibitor stabilize antibodies produced by plant roots. Plant Physiol. 141: 1185-1193

Laemmli UK (1970) Cleavage of structural proteins during the assembly of the head of bacteriophage T4. Nature 227: 680-685

Liénard D, Sourrouille C, Gomord V, Faye L (2007) Pharming and transgenic plants. Biotechnol. Annu. Rev. 13: 115-147

Lilley AK, Bailey MJ, Cartwright C, Turner SL, Hirsch PR (2006) Life in earth: the impact of GM plants on soil ecology? Trends Biotechnol. 24: 9-14

Munro S, Pelham HR (1987) A C-terminal signal prevents secretion of luminal ER proteins. Cell 48: 899-907

Murashige T, Skoog F (1962) A revised medium for rapid growth and bioassay with tobacco tissue cultures. Physiol. Plant. 15: 473-497

Petruccelli S, Otegui MS, Lareu F, Tran Dinh O, Fitchette AC, Circosta A, Rumbo M, Bardor M, Carcamo R, Gomord V, Beachy RN (2006) A KDEL-tagged monoclonal antibody is efficiently retained in the endoplasmic reticulum in leaves, but is both partially secreted and sorted to protein storage vacuoles in seed. Plant Biotechnol. J. 4: 511-527

Pujol M, Gavilondo J, Ayala M, Rodrìguez M, Gonzàlez EM, Pèrez L (2007) Fighting cancer with plant-expressed pharmaceuticals. Trends Biotechnol. 25: 455-459

Ramessar K, Peremerti A, Gomez-Galera S, Naqvi S, Moralejo M, Munoz P, Capell T, Christou P (2007) Biosafety and risk assessment framework for selectable marker genes in transgenic crop plants: a case of the science not supporting the politics. Transgenic Res. 16: 261-280

Sabharwal N, Icoz I, Saxena D, Stotzky G (2007) Release of the recombinant proteins, human serum albumin, betaglucuronidase, glycoprotein B from human cytomegalovirus, and green fluorescent protein, in root exudates from transgenic tobacco and their effects on microbes and enzymatic activities in soil. Plant Physiol. Biochem. 45: 464-469 
Saint-Jore-Dupas C, Faye L, Gomord V (2007) From planta to pharma with glycosylation in the toolbox. Trends Biotechnol. 25: 317-323

Sanvido O, Romeis J, Bigler F (2007) Ecological impacts of genetically modified crops: ten years of field research and commercial cultivation. Adv. Biochem. Eng. Biotechnol. 107: $235-278$

Saxena D, Flores S, Stotzky G (2002) Bt toxin is released in root exudates from 12 transgenic corn hybrids representing three transformation events. Soil Biol. Biochem. 34: 133-137

Saxena D, Stewart CN, Altosaar I, Shu Q, Stotzky G (2004) Larvicidal Cry proteins from Bacillus thuringensis are released in root exudates of transgenic B. thuringensis corn, potato and rice but not of $B$. thuringensis canola, cotton and tobacco. Plant Physiol. Biochem. 42: 383-387

Sparrow PAC, Irwin JA, Dale PJ, Twyman RM, Ma J-KC (2007) Pharma-Planta: road testing the developing regulatory guidelines for plant-made pharmaceuticals. Transgenic Res. 16: $147-161$

Spök A (2007) Molecular farming on the rise-GMO regulators still walking a tightrope. Trends Biotechnol. 25: 74-82

Streatfield SJ (2007) Approaches to achieve high-level heterologous protein production in plants. Plant Biotechnol. J. 5: $2-15$

Toubart P, Desiderio A, Salvi G, Cervone F, Daroda L, De Lorenzo G (1992) Cloning and characterization of the gene encoding the endopolygalacturonase-inhibiting protein (PGIP) of Phaseolus vulgaris L. Plant J. 2: 367-373
Towbin H, Staehelin T, Gordon J (1979) Electrophoretic transfer of proteins from polyacrylamide gels to nitrocellulose sheets: procedures and some applications. Proc. Natl. Acad. Sci. USA 76: 4350-4353

Twyman RM, Schillberg S, Fischer R (2005) Transgenic plants in the biopharmaceutical market. Expert Opin. Emerg. Drugs 10: 185-218

Van Droogenbroeck B, Cao J, Stadlmann J, Altmann F, Colanesi S, Hillmer S, Robinson DG, Van Lerberge E, Terryn N, van Montagu M, Liang M, Depicker A, De Jaeger G (2007) Aberrant localization and underglycosylation of highly accumulating single-chain Fv-Fc antibodies in transgenic Arabidopsis seeds. Proc. Natl. Acad. Sci. USA 104: $1430-1435$

van Esse HP, Thomma BPHJ, van't Klooster JW, de Wit PJGM (2006) Affinity-tags are removed from Cladosporium fulvum effector proteins expressed in the tomato leaf apoplast. J. Exp. Bot. 57: 599-608

Vitale A, Pedrazzini E (2005) Recombinant pharmaceuticals from plants: the plant endomembrane system as bioreactor. Mol. Interv. 5: 216-225

Widmer (2007) Assessing effects of transgenic crops on soil microbial communities. Adv. Biochem. Eng. Biotechnol. 107: 207-234

Wolt JD, Karaman S, Wang K (2007) Risk assessment for plant-made pharmaceuticals. CAB Reviews: Perspectives in Agriculture, Veterinary Science, Nutrition and Natural Resources 2: 1-9 